AIAA-98-4758

\title{
A COMPARISON OF APPROXIMATION MODELING TECHNIQUES: POLYNOMIAL VERSUS INTERPOLATING MODELS
}

\author{
Anthony A. Giunta* and Layne T. Watson ${ }^{\dagger}$ \\ Multidisciplinary Analysis and Design (MAD) Center for Advanced Vehicles \\ Virginia Polytechnic Institute and State University \\ Blacksburg, Virginia 24061
}

\begin{abstract}
Two methods of creating approximation models are compared through the calculation of the modeling accuracy on test problems involving one, five, and ten independent variables. Here, the test problems are representative of the modeling challenges typically encountered in realistic engineering optimization problems. The first approximation model is a quadratic polynomial created using the method of least squares. This type of polynomial model has seen considerable use in recent engineering optimization studies due to its computational simplicity and ease of use. However, quadratic polynomial models may be of limited accuracy when the response data to be modeled have multiple local extrema. The second approximation model employs an interpolation scheme known as kriging developed in the fields of spatial statistics and geostatistics. This class of interpolating model has the flexibility to model response data with multiple local extrema. However, this flexibility is obtained at an increase in computational expense and a decrease in ease of use. The intent of this study is to provide an initial exploration of the accuracy and modeling capabilities of these two approximation methods.
\end{abstract}

Keywords: approximation, response surface model, polynomial model, kriging, DACE

\section{Nomenclature}

\begin{tabular}{|c|c|}
\hline ANOVA & analysis of variance \\
\hline c & $\begin{array}{l}\text { vector of unknown coefficients in least } \\
\text { squares surface fitting }\end{array}$ \\
\hline$\hat{\mathbf{c}}$ & $\begin{array}{l}\text { vector of estimated coefficients in least } \\
\text { squares surface fitting }\end{array}$ \\
\hline DACE & $\begin{array}{l}\text { design and analysis of computer } \\
\text { experiments }\end{array}$ \\
\hline$f(\cdot)$ & unknown function \\
\hline$f(\cdot)$ & predicted function \\
\hline f & $\begin{array}{l}\text { vector of constants used in DACE } \\
\text { models }\end{array}$ \\
\hline MSE & mean squared error \\
\hline$n_{c}$ & number of candidate sample sites \\
\hline$n_{e}$ & $\begin{array}{l}\text { number of sample sites to calculate } \\
\text { modeling error }\end{array}$ \\
\hline$n_{s}$ & number of sample sites in design space \\
\hline$n_{t}$ & number of terms in a polynomial model \\
\hline$n_{v}$ & number of design variables \\
\hline $\mathbf{r}$ & vector of correlation values \\
\hline$R(\cdot)$ & correlation function \\
\hline $\mathbf{R}$ & correlation matrix \\
\hline RMS & root mean square error \\
\hline $\mathrm{RMS}_{\mathrm{ub}}$ & unbiased root mean square error \\
\hline RS & response surface \\
\hline RSM & response surface methodology \\
\hline$x$ & scalar component of $\mathbf{x}$ \\
\hline $\mathbf{x}$ & $\begin{array}{l}\text { vector denoting all locations in } \\
n_{v} \text {-dimensional space }\end{array}$ \\
\hline $\mathbf{x}^{(\mathbf{p})}$ & $\begin{array}{l}\text { vector denoting the } p^{t h} \text { location in } \\
n_{v} \text {-dimensional space }\end{array}$ \\
\hline
\end{tabular}

*Currently a National Research Council Postdoctoral Fellow, NASA Langley Research Center, MS 139 , Hampton, VA 23681. Member AIAA

$\dagger$ Professor of Computer Science and Mathematics

Copyright (C) 1998 by Anthony A. Giunta. Published by the American Institute of Aeronautics and Astronautics, Inc., with permission. 


\begin{tabular}{|c|c|}
\hline$\overline{\mathbf{x}}^{(\mathbf{p})}$ & $\begin{array}{l}\text { vector of the polynomial model terms } \\
\text { at the } p^{t h} \text { sample site }\end{array}$ \\
\hline$X$ & $\begin{array}{l}\text { matrix of sample site locations in least } \\
\text { squares surface fitting }\end{array}$ \\
\hline & scalar observed response value \\
\hline$y^{(p)}$ & $\begin{array}{l}\text { observed response value at the } p^{t h} \\
\text { sample site }\end{array}$ \\
\hline & mean of observed response values \\
\hline & scalar predicted response value \\
\hline$\hat{y}(\cdot)$ & prediction function \\
\hline 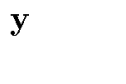 & $\begin{array}{l}\text { vector of observed response values at } \\
\text { sample sites }\end{array}$ \\
\hline & vector of predicted response values \\
\hline$Z(\cdot)$ & Gaussian random function \\
\hline$\beta$ & parameter in DACE modeling \\
\hline$\hat{\beta}$ & estimated DACE modeling parameter \\
\hline & deling error \\
\hline$\delta_{\max }$ & maximum modeling error \\
\hline$\delta_{\text {median }}$ & median modeling error \\
\hline 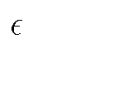 & $\begin{array}{l}\text { parameter used in defining the DACE } \\
\text { test function }\end{array}$ \\
\hline$\pi_{\beta}$ & prior distribution on $\beta$ \\
\hline & standard deviation of modeling errol \\
\hline$\sigma^{2}$ & sample variance \\
\hline$\hat{\sigma}^{2}$ & estimated sample variance \\
\hline 0 & $\begin{array}{l}\text { scalar correlation parameter used in } \\
\text { DACE modeling }\end{array}$ \\
\hline
\end{tabular}

\section{Introduction}

Approximation models are often used in engineering by others as well (cf., $[3,4,5,6,7,8,9])$.

Originally these polynomial modeling methods were developed to produce smooth approximation models of response data contaminated with random error found in typical physical (stochastic) experiments. Due to the ease of use of the polynomial modeling methods, these techniques migrated to the field of deterministic computer experiments where there is no random error (i.e., response data are identical each time the simulation is repeated). The applicability of using these methods in modeling deterministic response data is the subject of debate in the statistical community, some aspects of which are addressed by Simpson, et al [10].

In response to this issue, Sacks, et al [11] proposed the use of interpolation models to approximate response data obtained from deterministic computer simulations. Their interpolation models are based on techniques known as kriging originally developed in the fields of spatial statistics and geostatistics as described by Cressie in [12, pages 1-26] and [13]. (The term kriging was first used in the work of Matherton [14] who attributed the original development of the statistical techniques to mining engineer D. G. Krige.)

Recent studies by researchers at Boeing including Frank [15] and Booker [16] have employed DACE modeling methods in engineering optimization prob- rogates, has grown in popularity, a variety of model ing methods have been employed. Perhaps the most popular techniques involve polynomial models, typically linear or quadratic functions, created by performing a least squares curve fit to a set of data, where the data consist of one or more dependent response values along with one or more independent variables. Collectively, these polynomial-based modeling methods have come to be known as response surface models which is a term taken from the statistical literature (cf., Myers and Montgomery [1, pages 1-10]). These methods are popular for a number of reasons one of which is that they provide a compact and explicit functional relationship between the response and the independent variables. In addition, the method of least squares used in creating the models is relatively computationally inexpensive and straightforward. Evidence of the popularity of polynomial modeling is given by the wealth of recent reports both by the authors of this document [2] and trends whereas quadratic polynomials are by defini tion unimodal. However, the flexibility offered by DACE modeling methods is offset by the lack of an explicit model function as well as an increase in computational expense over that incurred in polynomial modeling.

The purpose of the research described here is to compare the modeling accuracy of both polynomial models and DACE models on a set of sample test problems. To the authors' knowledge, such a comparison has not been reported elsewhere and this investigation will provide useful quantitative and qualitative data on the utility of these modeling methods. In Section 2 the mathematical underpinnings of polynomial and DACE modeling are presented. Section 3 contains the description of the test problems and Section 4 contains an assessment of modeling accuracy for the test problems. A summary of this research is presented in Section 5. 


\section{Approximation Model For- mulation}

\subsection{Background on Approximation Models}

Prior to a description of the mathematical underpinnings of the approximation modeling methods, it is useful to compare the philosophy of polynomial modeling methods to that of DACE interpolating meth-

models based on Bayesian statistics and kriging. Although both RS models and DACE models are approximations to the true, unknown response surface and as such are technically response surface models, the statistical literature tends to reserve the term response surface model for polynomial models. The phrase polynomial RS model will be used to reinforce this distinction.

The construction of polynomial RS models or DACE interpolating models relies on the sampling of the design space at $n_{s}$ unique locations in the design space to obtain response values for the objective function or the constraints. Here, the design space is defined by the upper and lower bounds on the vector $\mathbf{x}$ of $n_{v}$ independent variables, where

$$
\mathbf{x}=\left[x_{1}, x_{2}, \ldots, x_{n_{v}}\right] .
$$

The upper and lower bounds create a design space in the shape of an $n_{v}$-dimensional cube which has $2^{n_{v}}$ vertices. Note that experimental error is not present when obtaining results from deterministic computer models. Thus, no information is gained from the repeated sampling of the same location in the design space. From the sampled data approximation models are constructed to describe the variation in the response(s) with respect to the $n_{v}$ independent variables. Mathematically, the true underlying functional relationship is expressed as

$$
y=f(\mathbf{x}),
$$

where $y$ is the observed response and $f(\mathbf{x})$ is the unknown function.

In many engineering optimization problems the cost of computing the objective function or constraints is computationally expensive. For this reason, approximation models are employed in the optimization problem as surrogates for these expensive function evaluations. These approximation models are expressed as

$$
\hat{y}=\hat{f}(\mathbf{x}) .
$$

Polynomial RS models can be thought of as "global" models in which all of the $n_{s}$ observed values of the response are equally weighted in the fitting of the polynomial surface. At an unsampled location in design space, $\mathbf{x}$, response observations that are near to $\mathbf{x}$ (in the sense of Euclidean distance) have an equal influence on the predicted response, $\hat{f}(\mathbf{x})$, as do the response observations that are far from $\mathbf{x}$. It may be argued that such a global model may not be the best approximator if the true unknown response has mul$\begin{array}{llllllll}\text { i l l l } & \text { l } & \text { i } & \text { h } & \text { i } & \text { i } & & \text { i }\end{array}$

less strongly influenced by those further away. Such local modeling behavior is characteristic of interpolation models, of which DACE models are one particular implementation.

\subsection{Polynomial Approximation Mod- els}

Polynomial response surface modeling (RSM) employs the statistical techniques of regression analysis and analysis of variance (ANOVA) to determine $\hat{f}(\mathbf{x})$ through a systematic decomposition of the variability in the observed response values. The empirical model is then estimated by assigning portions of the variability to either the effect of an independent variable or to random error.

If $n_{s}$ analyses are conducted and $p=1, \ldots, n_{s}$, then a quadratic response surface (RS) model has the form

$$
\begin{aligned}
y^{(p)}= & c_{o}+\sum_{1 \leq j \leq n_{v}} c_{j} x_{j}^{(p)}+ \\
& \sum_{1 \leq j \leq k \leq n_{v}} c_{\left(n_{v}-1+j+k\right)} x_{j}^{(p)} x_{k}^{(p)},
\end{aligned}
$$

where $y^{(p)}$ is the response; $x_{j}^{(p)}$ and $x_{k}^{(p)}$ are the $n_{v}$ design variables; and $c_{o}, c_{j}$, and $c_{\left(n_{v}-1+j+k\right)}$ are the unknown polynomial coefficients. Note that there are $n_{t}=\left(n_{v}+1\right)\left(n_{v}+2\right) / 2$ modeling terms in the quadratic polynomial. This polynomial model is written in matrix notation as

$$
y^{(p)}=\mathbf{c}^{T} \overline{\mathbf{x}}^{(\mathbf{p})},
$$

where $\mathbf{c}$ is the vector of length $n_{t}$ of unknown coefficients to be estimated,

$$
\mathbf{c}=\left[c_{0}, c_{1}, \ldots, c_{n_{t}-1}\right]
$$


$j \quad k$ model (Equation 4). For the $p^{\text {th }}$ observation this is

$$
\overline{\mathbf{x}}^{(\mathbf{p})}=\left[1, x_{1}^{(p)}, x_{2}^{(p)}, \cdots, x_{1}^{(p)} x_{2}^{(p)}, \cdots,\left(x_{n_{v}}^{(p)}\right)^{2}\right]
$$

h h i diff $\quad$ b $\quad h \quad t h$

Estimating the unknown coefficients requires $n_{s}$ analyses, where $n_{s} \geq n_{t}$. Under such conditions, the estimation problem is formulated in matrix notation as

$$
\mathbf{y} \approx \mathbf{X} \hat{\mathbf{c}}
$$

and $\mathbf{X}$ is the matrix formed by the $p$ row vectors $\overline{\mathbf{x}}^{(\mathbf{1})}, \ldots, \overline{\mathbf{x}}^{(\mathbf{p})}$ which is assumed to have rank $n_{t}$. Thus, $\mathbf{X}$ is expressed as

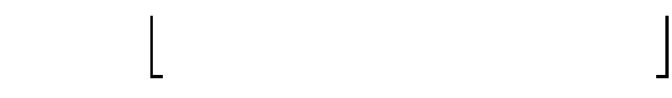
is

The unique least squares solution to Equation 8

$$
\hat{\mathbf{c}}=\left(\mathbf{X}^{T} \mathbf{X}\right)^{-1} \mathbf{X}^{T} \mathbf{y}
$$

this corresponds to

$$
\hat{\mathbf{y}}=\hat{\mathbf{c}}^{T} \overline{\mathbf{x}}
$$

Note that if $n_{s}>n_{t}$ the system of equations is overdetermined. Thus, the predicted response values (from the polynomial RS model) at the original sample locations may differ from the observed response values at the sampled locations.

\subsection{DACE Approximation Models}

The objective here is to provide an introduction to the statistics and mathematics of DACE modeling. A detailed treatment of the statistical and mathematical methods involved in DACE modeling is found in the work of Sacks et al. [11]; Koehler and Owen [17]; Osio and Amon [18]; and Booker et al. [19].

Before addressing the principles underlying DACE modeling methods, it is useful at this point to to the probability density function which one assigns to a variable of unknown value before any experimental data on that variable are collected [20, pages 4,5$]$. The prior distribution is the mechanism in Bayesian

This intentional bias is the source of much controversy in the statistical community. In spite of the differences between classical statistics and Bayesian statistics, Berger [20, pages 109,110] emphasizes that both classical and Bayesian statistics have merit and

In the DACE literature the true, unknown function to be modeled is typically expressed as

$$
y(\mathbf{x})=f(\mathbf{x})+Z(\mathbf{x})
$$

\begin{abstract}
) ( )
Equation 13 in some sense is a "global" model for the entire design space based on the $n_{s}$ response observations, while the $Z(\mathbf{x})$ term creates a "localized" deviation from the global model.
\end{abstract}

$$
y(\mathbf{x})=\beta+Z(\mathbf{x})
$$

The term $\beta$ takes on different meanings depending on one's statistical point of view. From the perspective of the kriging approach used in DACE, $\beta$ is an unknown constant to be estimated based on the $n_{s}$ observed response values. From the perspective of Bayesian statistics $\beta$ is a random variable with a prior distribution denoted as $\pi_{\beta}$. The interpretations of $\beta$ are identical regardless of the statistical perspective if $Z(\mathbf{x})$ has a Gaussian distribution and $\pi_{\beta}$ has a uniform distribution [11], i.e., if no prior knowledge is used to provide an initial estimate for $\beta$.

The covariance matrix of $Z(\mathbf{x})$ is expressed as

$$
\operatorname{Cov}\left[Z\left(\mathbf{x}^{(i)}\right), Z\left(\mathbf{x}^{(j)}\right)\right]=\sigma^{2} \mathbf{R}\left[R\left(\mathbf{x}^{(i)}, \mathbf{x}^{(j)}\right)\right]
$$

where $\mathbf{R}$ is the correlation matrix, and $R$ is the correlation function which is selected by the user. In 
Equation $15 i=1, \ldots, n_{s}$ and $j=1, \ldots, n_{s}$. Note that the correlation matrix $\mathbf{R}$ is symmetric with values of unity along the diagonal.

As noted above, the user may select the form of the correlation function $R$. Sacks et al. [11]; Koehler and Owen [17]; and Booker et al. [19] provide a detailed description of various correlation functions that may be used. A choice for $R$ often found in the statistical literature, and employed in [19], is an exponential correlation function

$$
R\left(\mathbf{x}^{(i)}, \mathbf{x}^{(j)}\right)=\exp \left[-\sum_{k=1}^{n_{v}} \theta_{k}\left|\mathbf{x}_{k}^{(i)}-\mathbf{x}_{k}^{(j)}\right|^{2}\right],
$$

where $\theta_{k}$ is the vector of unknown correlation parameters. For this research only a single correlation parameter is used instead of a vector of correlation parameters. The scalar correlation parameter is denoted as $\theta$. Thus, Equation 16 may be rewritten as

$$
R\left(\mathbf{x}^{(i)}, \mathbf{x}^{(j)}\right)=\exp \left[-\theta \sum_{k=1}^{n_{v}}\left|\mathbf{x}_{k}^{(i)}-\mathbf{x}_{k}^{(j)}\right|^{2}\right],
$$

The process by which a value for $\theta$ is estimated is given below.

Another term of interest is the correlation vector, $\mathbf{r}(\mathbf{x})$, between the response at a location, $\mathbf{x}$, and the $\mathbf{x}^{(1)}, \ldots, \mathbf{x}^{\left(n_{s}\right)}$ response values. The correlation vector is expressed as

$$
\begin{aligned}
\mathbf{r}(\mathbf{x}) & =R\left(\mathbf{x}, \mathbf{x}^{(i)}\right) \\
& =\left[R\left(\mathbf{x}, \mathbf{x}^{(1)}\right), R\left(\mathbf{x}, \mathbf{x}^{(2)}\right), \cdots, R\left(\mathbf{x}, \mathbf{x}^{\left(n_{s}\right)}\right)\right]
\end{aligned}
$$

While Equation 14 represents the true, unknown function to be approximated, the computed (i.e., estimated) DACE model is given the symbol $\hat{y}(\mathbf{x})$. In statistical notation, this estimated DACE model is defined as

$$
\hat{y}(\mathbf{x})=E\left(y(\mathbf{x}) \mid y\left(\mathbf{x}^{(1)}\right), \ldots, y\left(\mathbf{x}^{\left(n_{s}\right)}\right)\right),
$$

where the expression $E(\cdot)$ is the statistical symbol for the expected value of $(\cdot)$ and the expression $E(A \mid B)$ is the expected value of $A$ given the information $B$. The terms $y\left(\mathbf{x}^{(1)}\right), \ldots, y\left(\mathbf{x}^{\left(n_{s}\right)}\right)$ are the $n_{s}$ observed values of the response, $y(\mathbf{x})$ is the true response one is attempting to estimate, and $\hat{y}(\mathbf{x})$ is the actual estimate of the response (which one hopes is close to $y(\mathbf{x})$ ). This distinction between $\hat{y}(\mathbf{x})$ and $y(\mathbf{x})$ is necessary so that the concept of mean squared error (MSE) may be introduced where

$$
M S E=E(\hat{y}(\mathbf{x})-y(\mathbf{x}))^{2} .
$$

This is simply a measure of the amount of error between the DACE model, $\hat{y}(\mathbf{x})$, and the true model, $y(\mathbf{x})$, at all locations, $\mathbf{x}$, in the design space. Since the DACE model performs interpolation there is no error between the DACE model and the true model at the $n_{s}$ sites where the values of the response are known.

If MSE is minimized, $\hat{y}(\mathbf{x})$ becomes

$$
y(\mathbf{x})=\hat{\beta}+\mathbf{r}^{T}(\mathbf{x}) \mathbf{R}^{-1}(\mathbf{y}-\hat{\beta} \mathbf{f}),
$$

where $\hat{\beta}$ is unknown, and both $\mathbf{r}(\mathbf{x})$ and $\mathbf{R}$ depend on the unknown parameter $\theta$. Note that the vector $\mathbf{f}$ has length $n_{s}$ with all entries equal to unity

$$
\mathbf{f}=[1, \ldots, 1],
$$

which is a result of the assumption that all of the variability in $y(\mathbf{x})$ is accounted for in the $Z(\mathbf{x})$ term. While the usual notation for a vector with all entries equal to unity is $\mathbf{e}$, the vector $\mathbf{f}$ is retained to maintain similarity with the notation used in Koehler and Owen [17] and Booker et al. [19].

The unknown parameter $\theta$ is found using maximum likelihood estimation as described by Booker et al. [19]. In this approach, the values for $\hat{\beta}$ and the estimated variance, $\hat{\sigma}^{2}$, are obtained using generalized least squares as

$$
\hat{\beta}=\left(\mathbf{f}^{T} \mathbf{R}^{-1} \mathbf{f}\right)^{-1} \mathbf{f}^{T} \mathbf{R}^{-1} \mathbf{y},
$$

and

$$
\hat{\sigma}^{2}=\frac{(\mathbf{y}-\hat{\beta} \mathbf{f})^{T} R^{-1}(\mathbf{y}-\hat{\beta} \mathbf{f})}{n_{s}} .
$$

Note that Equations 23 and 24 implicitly depend on the correlation parameter $\theta$.

The maximum likelihood estimation of $\theta$ is reduced to a one-dimensional optimization problem with simple bounds of the form

$$
\begin{array}{rc}
\max _{\theta \in R^{1}} & (-1 / 2)\left[\left(n_{s} \ln \hat{\sigma}^{2}\right)+\ln |\mathbf{R}|\right], \\
\text { subject to } & 0 \leq \theta \leq \infty .
\end{array}
$$

Thus, by solving this one-dimensional optimization problem the DACE approximation model $\hat{y}(\mathbf{x})$ is completely defined. Note that if Equation 16 were used (i.e., retaining a vector of correlation parameters), then the one-dimensional minimization problem becomes an $n_{v}$-dimensional minimization problem. 


\section{Approximation Model Test Problems}

The objective of performing the test problems was to gain an understanding of the strengths and weaknesses of DACE modeling as compared to polynomial RS modeling. For these efforts two test problems were formulated where the first test problem was expected to be biased in favor of the DACE modeling method and the second test problem was expected to be biased in favor of the polynomial RS modeling method. A critical element of this comparison is the investigation of how the accuracy of the DACE models and RS models is affected as the number of dimensions, $n_{v}$, increases. To investigate this aspect of modeling accuracy, test problems involving $n_{v}=1$, $n_{v}=5$, and $n_{v}=10$ were examined.

\subsection{Test Problem Formulation}

For this investigation a simple test function was chosen so that it could be exhaustively examined with minimal computational expense. This test function has the form

$$
y(\mathbf{x})=\sum_{i=1}^{n_{v}}\left[\frac{3}{10}+\sin \left(\frac{16}{15} x_{i}-\epsilon\right)+\sin ^{2}\left(\frac{16}{15} x_{i}-\epsilon\right)\right],
$$

where the term $\epsilon$ acts as a shifting mechanism to make the response, $y(\mathbf{x})$, appear more or less quadratic on the range $[-1,1]^{n_{v}}$. The values used for $\epsilon$ are described below. Since there is no numerical noise inherent in Equation 26 it is henceforth referred to as the smooth test function.

To simulate the effects of numerical noise often encountered in realistic engineering optimization problems (cf., [2], [21], [22], [23], [24]), a high-frequency low-amplitude sine wave function was added to Equation 26 . This noisy test function has the form

$$
\begin{aligned}
y(\mathbf{x})= & \sum_{i=1}^{n_{v}}\left[\frac{3}{10}+\sin \left(\frac{16}{15} x_{i}-\epsilon\right)+\sin ^{2}\left(\frac{16}{15} x_{i}-\epsilon\right)+\right. \\
& \left.\frac{2}{100} \sin \left(40\left(\frac{16}{15} x_{i}-\epsilon\right)\right)\right],
\end{aligned}
$$

where the term on the far right of Equation 27 is the high frequency, low amplitude component.

The first test function (Case 1) was created for $\epsilon=1.0$ and a plot of the noisy version of this function for $n_{v}=1$ is shown in Figure 1. Both the smooth and noisy variants of the Case 1 test functions are shown in Figure 2 for $n_{v}=2$. This function appears quasi-sinusoidal on $[-1,1]$.
The Case 2 test function was created using $\epsilon=0.7$ and has a quasi-quadratic trend on $[-1,1]$. The noisy Case 2 test function is shown in Figure 3 for $n_{v}=1$ and both the smooth and noisy Case 2 test functions are shown in figure 4 for $n_{v}=2$.

\subsection{Evaluation of Modeling Accuracy}

For both Cases 1 and 2, DACE and RS models (denoted as $\hat{y}(\mathbf{x}))$ were constructed based on $n_{s}$ evaluations (response values) of the noisy test function. These models were then used to estimate the unknown response values of the smooth test function at $n_{e}$ locations, where $n_{e} \gg n_{s}$. These predicted smooth function response values are denoted as $\hat{y}_{n_{e}}$. To evaluate the accuracy of the DACE and RS models, the actual response values of the smooth test function are also calculated for the $n_{e}$ locations. These actual smooth function response values are denoted as $y_{n_{e}}$. The discrepancy between $y_{n_{e}}$ and $\hat{y}_{n_{e}}$ is known as the modeling error. Note that the definition of the modeling error is different from the residual error which is the discrepancy between a polynomial model and the data points in an overdetermined least squares problem. There is no residual error in DACE modeling since the DACE method exactly interpolates the $n_{s}$ response values.

The total modeling error in the DACE and RS models is characterized using five error metrics. These are the mean error, $\bar{\delta}$, the median error, $\delta_{\text {median }}$, the standard deviation, $\sigma_{\delta}$, the maximum error, $\delta_{\max }$, and the unbiased RMS error RMS $\mathrm{Rub}_{\mathrm{ub}}$.

In these error metrics the modeling error is defined as

$$
\delta_{i}=\left|\hat{y}_{i}-y_{i}\right|,
$$

for $i=1, \ldots, n_{e}$. Using this notation, the mean modeling error is

$$
\bar{\delta}=\frac{1}{n_{e}} \sum_{i=1}^{n_{e}} \delta_{i},
$$

and the standard deviation of the modeling error is

$$
\sigma_{\delta}=\sqrt{\frac{\sum_{i=1}^{n_{e}}\left(\delta_{i}-\bar{\delta}\right)^{2}}{n_{e}-1}} .
$$

The median modeling error, $\delta_{\text {median }}$, is defined as the midpoint value of the series in which the $\delta_{i}$ values are placed in ascending value. The maximum value of this series is the maximum modeling error, $\delta_{\max }$, which is defined as

$$
\delta_{\max }=\max \left(\delta_{i}\right)
$$


In addition to these metrics, the root mean squared modeling error is

$$
\operatorname{RMS}=\sqrt{\frac{\sum_{i=1}^{n_{e}} \delta_{i}{ }^{2}}{n_{e}}} .
$$

If the $n_{e}$ locations are not the same as the sample sites, $n_{s}$, used to construct the approximation model, then Equation 32 is an unbiased estimator of the RMS modeling error and is identified as $\mathrm{RMS}_{\mathrm{ub}}$. However, if $n_{e}$ and $n_{s}$ are the same, then Equation 32 is biased and it underestimates the true error. When $n_{e}$ and $n_{s}$ are the same the unbiased RMS error must be calculated using

$$
\mathrm{RMS}_{\mathrm{ub}}=\sqrt{\frac{\sum_{i=1}^{n_{e}} \delta_{i}{ }^{2}}{n_{e}-n_{t}}},
$$

where $n_{t}$ is the number of terms in the polynomial model. See Myers and Montgomery [1, page 26] for more information on unbiased estimators.

\section{Results}

\subsection{One Variable Test Problem}

In the one variable test problem the test functions were sampled at three locations $\left(n_{s}=3\right)-0.5,-0.3$, and 0.7 on $[-1,1]$. From the response values at these three sites a DACE model and a quadratic polynomial RS model were created. To test the accuracy of the DACE and RS models, the smooth test function was sampled at 201 equally spaced points along $[-1,1]$. Figures 5 and 6 show the DACE and RS models used in Cases 1 and 2, respectively.

For the Case 1 test problem the DACE model had a correlation parameter of $\theta=7.540$ and $\hat{\beta}=0.2127$, while for the Case 2 test problem these values were $\theta=28.394$ and $\hat{\beta}=0.2777$. The quadratic response surface polynomial models for both the Case 1 and Case 2 test functions were created using the Fit[.] function in Mathematica [25, pages 859-861].

In addition to a DACE model and a quadratic polynomial RS model a third model was examined where

$$
\hat{y}(\mathbf{x})=\bar{y}
$$

where $\bar{y}$ is the mean of the $n_{s}$ observed response values in $\mathbf{y}$. This mean-value model was selected since it represents what is perhaps the most simple, computationally inexpensive, approximation model one may create. Further, it provides a sort of lower bound on modeling accuracy, i.e., one would expect a more "complex" approximation model would be at least as accurate as the mean-value model. In addition, the DACE interpolation model has the property that it becomes a mean-value model when it is used to interpolate far from any sample sites. Note that in the one variable test problem the mean-value models are $\bar{y}=0.2342$ for Case 1 and $\bar{y}=0.2680$ for Case 2 .

The modeling errors for DACE, polynomial RS, and mean-values models were calculated using the 201 values of the smooth test function. The results for Cases 1 and 2 are shown in Table 1 . For Case 1 the DACE model is more accurate than both the polynomial RS model and the mean-value model, whereas for Case 2 the polynomial RS model is more accurate than the other two models. This corresponds to the trends shown in Figures 5 and 6.

\subsection{Five Variable Test Problem}

For the five variable test problem $n_{s}=50$ and $n_{e}=3125$. The 50 sample sites correspond to those obtained from a $D$-optimal experimental design used in previous research related to this work (see Giunta, et al [2]), with all of the sample sites contained in the domain defined by $[-1,1]^{5}$. The 3125 test sites were created by discretizing the design space into a $5 \times 5 \times 5 \times 5 \times 5$ mesh where $5^{5}=3125$.

For the Case 1 test problem the DACE model had a correlation parameter of $\theta=0.45$ and $\hat{\beta}=1.3516$, while for the Case 2 test problem these values were $\theta=0.08$ and $\hat{\beta}=5.9593$. As above, the quadratic response surface polynomial models for the Case 1 and Case 2 test functions were created using the Fit[.] function in Mathematica.

In addition to a DACE model and a quadratic polynomial RS model, two other approximation methods were examined. The third model is a combined RS/DACE model of the form

$$
\hat{y}(\mathbf{x})=f(\mathbf{x})+\beta_{\text {residual }}+Z(\text { residual }),
$$

where $f(\mathbf{x})$ is the quadratic polynomial RS model found using Mathematica and $\beta_{\text {residual }}+Z$ (residual) is a DACE model applied to the residual error existing in the least squares surface fit for $f(\mathbf{x})$. For the Case 1 RS/DACE model the optimal correlation parameter was $\theta=30.0$, and $\hat{\beta}=-5.25 \cdot 10^{-7}$. In the RS/DACE model for Case 2 these parameters were $\theta=30.0$, and $\hat{\beta}=-5.76 \cdot 10^{-7}$. The fourth model examined is the mean-value model (Equation 34) where for Case $1, \bar{y}=1.2512$ and for Case $2, \bar{y}=2.1418$.

The modeling errors for these four approximation models were calculated for Case 1 and Case 2 test functions and are listed in Table 2. In the Case 1 results the polynomial $\mathrm{RS}$ model and the combined RS/DACE model have nearly identical values for the 
modeling errors. For the DACE method the modeling error is not as low as for the polynomial-based models, but it is lower than for the mean-value model. Similar trends are exhibited in the Case 2 results where the modeling errors for the polynomial RS model and the RS/DACE model are nearly identical, are the modeling errors for the DACE model are only marginally worse. In Case 2 however, the meanvalue model has considerable higher modeling errors than the other three models.

\subsection{Ten Variable Test Problem}

For the ten variable test problem $n_{s}=132$ and $n_{e}=10000$. The 132 sample sites were obtained from a D-optimal experimental design used in previous work by Giunta, et al [2] and were located within the ten dimensional design space defined by $\left[\begin{array}{ll}-1 & 1\end{array}\right]^{10}$ The

variable test problem were the same as those used in the five variable test problem. For the Case 1 test problem the DACE model had a correlation parameter of $\theta=0.50$ and $\hat{\beta}=2.6455$, while for the Case 2 test problem these values were $\theta=0.50$ and $\hat{\beta}=4.7987$. As before, the quadratic response surface polynomial models for both the Case 1 and Case 2 test functions were created using the Fit[.] function in Mathematica. For the Case $1 \mathrm{RS} / \mathrm{DACE}$ model the optimal correlation parameter was $\theta=0.10$, and $\hat{\beta}=4.69 \cdot 10^{-15}$, and for Case 2 , these parameters were $\theta=0.10$, and $\hat{\beta}=7.31 \cdot 10^{-15}$. For the fourth model, Case 1 was $\bar{y}=2.6214$ and Case 2 was $\bar{y}=4.7190$.

The results for the Case 1 and Case 2 test problems are listed in Table 3 . In Case 1, the polynomial RS model and the RS/DACE model exhibit nearly identical modeling errors and provide the best approximations to the test function. The modeling error for the DACE model is somewhat worse than for the polynomial-based models, and the modeling error for the mean-value model is the largest.

While the results for the Case 1 test problem are similar for the five and ten variable versions of the test problem, this is not true for the Case 2 test problem.

\subsection{Summary of Test Problem Results}

Note that some caution must be exercised in interpreting these results as the modeling accuracy data and observations are applicable only to the Case 1 and Case 2 test functions considered here. As may be expected, if different test functions had been investigated, the results may have been different. In fact it is quite easy to create a test function for which the mean-value model is the most accurate modeling method, as the authors discovered in some initial DACE modeling work.

The results from the one variable test problem showed the expected trends, i.e., where the DACE model was more accurate for the Case 1 test function and the polynomial RS model was more accurate for the Case 2 test function. However, the five and ten variable versions of the Case 1 test problem did $t$ i ld th $t d$ lt $F$ th i

the DACE model were only marginally more accurate than the mean-value model. Thus, the sinusoidal features of the test problem posed difficulties for both the polynomial RS and DACE models.

For the one, five, and ten variable versions of the Case 2 test problem, it is clear that the polynomial RS model provides the highest modeling accuracy of the approximation methods considered in this study. These results were expected since the test function is quasi-quadratic. However, the most startling results are shown in the modeling error data for the DACE model as compared to the mean-value model for the ten variable test problem. Here, the DACE model is only slightly more accurate than the mean-value model.

\section{Conclusions}

In this study, the accuracy of quadratic polynomial models and DACE interpolating models was evaluated through the examination of several test problems. The data obtained in this study showed that the quadratic polynomial models were more accurate, the DACE model. Note that once again the DACE model is only slightly more accurate than the meanvalue model. sory investigation is not intended to serve as an exhaustive comparison between the two modeling methods. 


\section{Future Work}

Clearly, there are numerous opportunities for further investigation, in both the formulation of the DACE models and in the examination of other test problems. For the test cases described in this study, future areas of investigation include (1) the use of a vector of correlation parameters in the exponential correlation model, and (2) the examination of various methods to select sample sites in the design space. Both of these may significantly affect the modeling accuracy of DACE approximation models.

\section{Acknowledgements}

The authors wish to thank Prof. Bernard Grossman and Prof. William H. Mason of Virginia Tech, and Prof. Raphael T. Haftka of the University of Florida for their participation in this research effort. In addition, the authors are grateful for the comments and suggestions from Dr. James R. Koehler and Dr. Andrew J. Booker.

This work was supported by NASA Grant NAG1-1562 while the first author was a graduate student in the Department of Aerospace and Ocean Engineering at Virginia Tech.

\section{References}

[1] Myers, R. H. and Montgomery, D. C. Response Surface Methodology: Process and Product Optimization Using Designed Experiments, pp. 1141, 279-401, 462-480, John Wiley \& Sons, Inc., New York, NY (1995).

a. Supersonic Transport Using Design of Experiments Theory and Response Surface Modelling," Aeronautical J., 101(1008), 347-356 (1997).

[3] Healy, M. J., Kowalik, J. S., and Ramsay, J. W.

[4] Engelund, W. C., Stanley, D. O., Lepsch, R. A., McMillin, M. M., and Unal, R. "Aerodynamic Configuration Design Using Response Surface Methodology Analysis," AIAA Paper 93-3967 (1993)
Method to the Design of Tipjet Driven Stopped Rotor/Wing Concepts," AIAA Paper 95-3965 (1995).

[6] Chen, W., Allen, J. K., Schrage, D. P., and Mistree, F. "Statistical Experimentation Methods for Achieving Affordable Concurrent Systems Design," AIAA J., 35(5), 893-900 (1997).

[7] Sellar, R. S., Stelmack, M. A., Batill, S. M., and Renaud, J. E. "Response Surface Approximations for Discipline Coordination in Multidisciplinary Design Optimization," AIAA Paper 961383 (1996).

[8] Roux, W. J., Stander, N., and Haftka, R. T. "Response Surface Approximations for Structural Optimization," in Proceedings of the 6th AIAA/NASA/ISSMO Symposium on Multidisciplinary Analysis and Optimization, pp. 565578, Bellevue, WA, AIAA Paper 96-4042 (Sept. 1996).

[9] Toropov, V. V. "Simulation Approach to Structural Optimization," Structural Optimization, 1, 37-46 (1989).

[10] Simpson, T. W., Pepliski, J., Koch, P. N., and Allen, J. K. "On the Use of Statistics in Design and the Implications for Deterministic Computer Experiments," in Design Theory and Methodology - DTM'C97, Sacramento, CA, Paper No. DETC97/DTM-3881 (Sept. 1997).

[11] Sacks, J., Welch, W. J., Mitchell, T. J., and Wynn, H. P. "Design and Analysis of Computer

1-26, John Wiley \& Sons, Inc., New York, NY (1991).

[13] Cressie, N. "Geostatistics," The American St ti ti $i \quad 43(4) 197202(\mathrm{~N} \quad$ b 1989)

[15] Frank, P. "Global Modeling for Optimization," SIAG/OPT Views-and-News, 7, 9-12 (1995).

[16] Booker, A. J. "Case Studies in Design and Anal- 
[17] Koehler, J. R. and Owen, A. B. Computer Experiments, volume 13 of Handbook of Statistics, pp. 261-308, Elsevier Science, New York, NY, eds. S. Ghosh and C. R. Rao (1996).

[18] Osio, I. G. and Amon, C. H. "An Engineering Design Methodology with Multistage Bayesian Surrogates and Optimal Sampling," Research in Engineering Design, 8, 189-206 (1996).

[19] Booker, A. J., Conn, A. R., Dennis, J. E., Frank, P. D., Trosset, M., and Torczon, V. "Global Modeling for Optimization: Boeing/IBM/Rice Collaborative Project," Text provided by Paul D. Frank of Boeing (1995).

[20] Berger, J. O. Statistical Decision Theory and Bayesian Analysis, pp. 4,5,109,110, SpringerVerlag, New York, NY (1985).

[21] Venter, G., Haftka, R. T., and Starnes, J. H. "Construction of Response Surfaces for Design Optimization Applications," in Proceedings of the 6th AIAA/NASA/ISSMO Sympo- sium on Multidisciplinary Analysis and Optimization, pp. 548-564, Bellevue, WA, AIAA Paper 96-4040 (Sept. 1996).

[22] Kaufman, M., Balabanov, V., Burgee, S. L., Giunta, A. A., Grossman, B., T., H. R., H., M. W., and Watson, L. T. "VariableComplexity Response Surface Approximations for Wing Structural Weight in HSCT Design," J. Computational Mechanics, 18(2), 112-126 (1996).

[23] Narducci, R., Grossman, B., and Haftka, R. T. "Sensitivity Algorithms for Inverse Design Problems Involving Shock Waves," Inverse Problems in Engineering, 2, 49-83 (1995).

[24] Wu, X., Cliff, E. M., and Gunzburger, M. D. "An Optimal Design Problem for a Two-Dimensional Flow in a Duct," Optimal Control Applications 6 Methods, 17, 329-339 (1996).

[25] Wolfram, S. The Mathematica Book, Champaign, IL, 3rd edition (1996). 
Table 1: Modeling errors in Cases 1 and 2 for the one variable test problem.

\begin{tabular}{cccccc} 
Model & $\begin{array}{c}\text { Mean } \\
\text { Error }\end{array}$ & $\begin{array}{c}\text { Median } \\
\text { Error }\end{array}$ & $\begin{array}{c}\text { Std. } \\
\text { Dev. }\end{array}$ & $\begin{array}{c}\mathbf{R M S}_{\mathbf{u b}} \\
\text { Error }\end{array}$ & $\begin{array}{c}\text { Max. } \\
\text { Error }\end{array}$ \\
\hline \hline Case $\mathbf{1}(\epsilon=1.0)$ & & & & & \\
\hline$\hat{y}(\mathbf{x})=\beta+Z(\mathbf{x})$ & 0.051 & 0.027 & 0.045 & 0.068 & 0.203 \\
$\hat{y}(\mathbf{x})=\hat{\mathbf{c}}^{T} \overline{\mathbf{x}}$ & 0.076 & 0.057 & 0.076 & 0.107 & 0.328 \\
$\hat{y}(\mathbf{x})=\bar{y}$ & 0.081 & 0.063 & 0.058 & 0.100 & 0.184 \\
\hline Case $\mathbf{2}(\epsilon=0.7)$ & & & & & \\
\hline$\hat{y}(\mathbf{x})=\beta+Z(\mathbf{x})$ & 0.115 & 0.093 & 0.112 & 0.160 & 0.502 \\
$\hat{y}(\mathbf{x})=\hat{\mathbf{c}}^{T} \overline{\mathbf{x}}$ & 0.080 & 0.065 & 0.081 & 0.114 & 0.393 \\
$\hat{y}(\mathbf{x})=\bar{y}$ & 0.130 & 0.117 & 0.110 & 0.170 & 0.519
\end{tabular}

Table 2: Modeling errors in Cases 1 and 2 for the five variable test problem.

\begin{tabular}{cccccc} 
Model & $\begin{array}{c}\text { Mean } \\
\text { Error }\end{array}$ & $\begin{array}{c}\text { Median } \\
\text { Error }\end{array}$ & $\begin{array}{c}\text { Std. } \\
\text { Dev. }\end{array}$ & $\begin{array}{c}\text { RMS }_{\mathbf{u b}} \\
\text { Error }\end{array}$ & $\begin{array}{c}\text { Max. } \\
\text { Error }\end{array}$ \\
\hline \hline Case $\mathbf{1}(\epsilon=1.0)$ & & & & & \\
\hline$\hat{y}(\mathbf{x})=\beta+Z(\mathbf{x})$ & 0.211 & 0.180 & 0.159 & 0.264 & 0.992 \\
$\hat{y}(\mathbf{x})=\hat{\mathbf{c}}^{T} \overline{\mathbf{x}}$ & 0.202 & 0.171 & 0.154 & 0.254 & 0.963 \\
$\hat{y}(\mathbf{x})=f(\mathbf{x})+\beta_{\text {res. }}+Z($ res. $)$ & 0.203 & 0.181 & 0.153 & 0.254 & 0.963 \\
$\hat{y}(\mathbf{x})=\bar{y}$ & 0.241 & 0.210 & 0.175 & 0.298 & 0.989 \\
\hline Case $\mathbf{2}(\epsilon=0.7)$ & & & & & \\
\hline$\hat{y}(\mathbf{x})=\beta+Z(\mathbf{x})$ & 0.225 & 0.190 & 0.171 & 0.282 & 0.945 \\
$\hat{y}(\mathbf{x})=\hat{\mathbf{c}}^{T} \mathbf{\mathbf { x }}$ & 0.210 & 0.178 & 0.158 & 0.263 & 0.944 \\
$\hat{y}(\mathbf{x})=f(\mathbf{x})+\beta_{\text {res. }}+Z$ (res. $)$ & 0.211 & 0.179 & 0.158 & 0.264 & 0.944 \\
$\hat{y}(\mathbf{x})=\bar{y}$ & 0.696 & 0.651 & 0.425 & 0.815 & 1.793
\end{tabular}

Table 3: Modeling errors in Cases 1 and 2 for the ten variable test problem.

\begin{tabular}{cccccc} 
Model & $\begin{array}{c}\text { Mean } \\
\text { Error }\end{array}$ & $\begin{array}{c}\text { Median } \\
\text { Error }\end{array}$ & $\begin{array}{c}\text { Std. } \\
\text { Dev. }\end{array}$ & $\begin{array}{c}\text { RMS } \\
\text { Error }\end{array}$ & $\begin{array}{c}\text { Max. } \\
\text { Error }\end{array}$ \\
\hline \hline Case $\mathbf{1}(\epsilon=1.0)$ & & & & & \\
\hline$\hat{y}(\mathbf{x})=\beta+Z(\mathbf{x})$ & 0.651 & 0.636 & 0.362 & 0.745 & 2.010 \\
$\hat{y}(\mathbf{x})=\hat{\mathbf{c}}^{T} \overline{\mathbf{x}}$ & 0.524 & 0.477 & 0.355 & 0.633 & 1.964 \\
$\hat{y}(\mathbf{x})=f(\mathbf{x})+\beta_{\text {res }}+Z($ res. $)$ & 0.524 & 0.479 & 0.348 & 0.629 & 1.823 \\
$\hat{y}(\mathbf{x})=\bar{y}$ & 0.698 & 0.696 & 0.283 & 0.753 & 1.801 \\
\hline Case $\mathbf{2}(\epsilon=0.7)$ & & & & & \\
\hline$\hat{y}(\mathbf{x})=\beta+Z(\mathbf{x})$ & 2.090 & 1.920 & 0.531 & 2.157 & 4.071 \\
$\hat{y}(\mathbf{x})=\hat{\mathbf{c}}^{T} \overline{\mathbf{x}}$ & 0.380 & 0.326 & 0.218 & 0.473 & 1.646 \\
$\hat{y}(\mathbf{x})=f(\mathbf{x})+\beta_{\text {res. }}+Z($ res. $)$ & 0.544 & 0.475 & 0.416 & 0.693 & 2.566 \\
$\hat{y}(\mathbf{x})=\bar{y}$ & 2.344 & 2.385 & 0.528 & 2.403 & 3.914
\end{tabular}




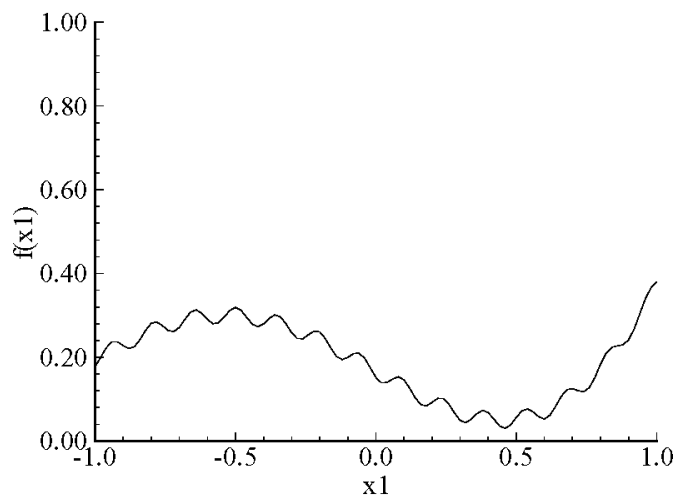

Figure 1: A one dimensional view of the Case 1 test function $(\epsilon=1.0)$.
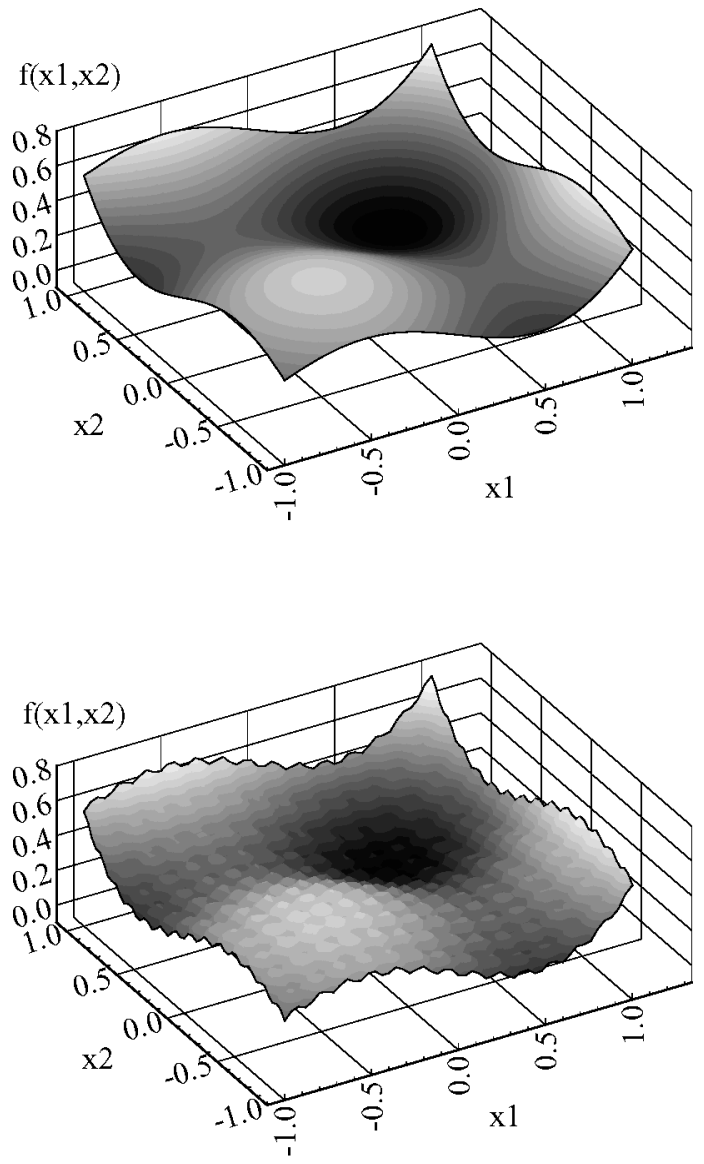

Figure 2: A two dimensional view of the smooth (top) and noisy variants of the Case 1 test function $(\epsilon=$ $1.0)$.

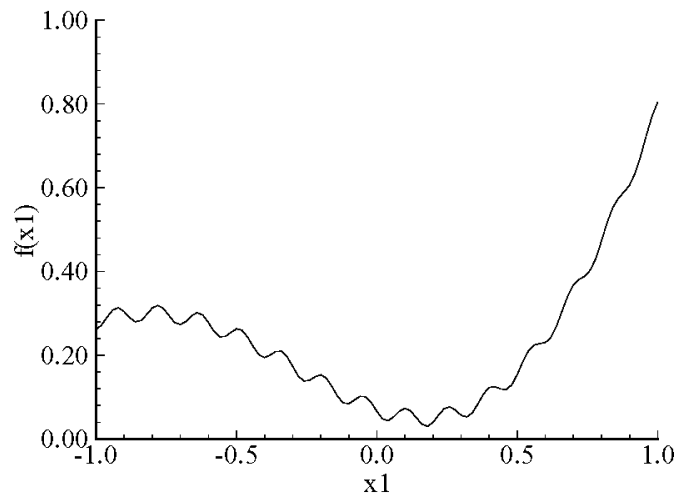

Figure 3: A one dimensional view of the Case 2 test function $(\epsilon=0.7)$.
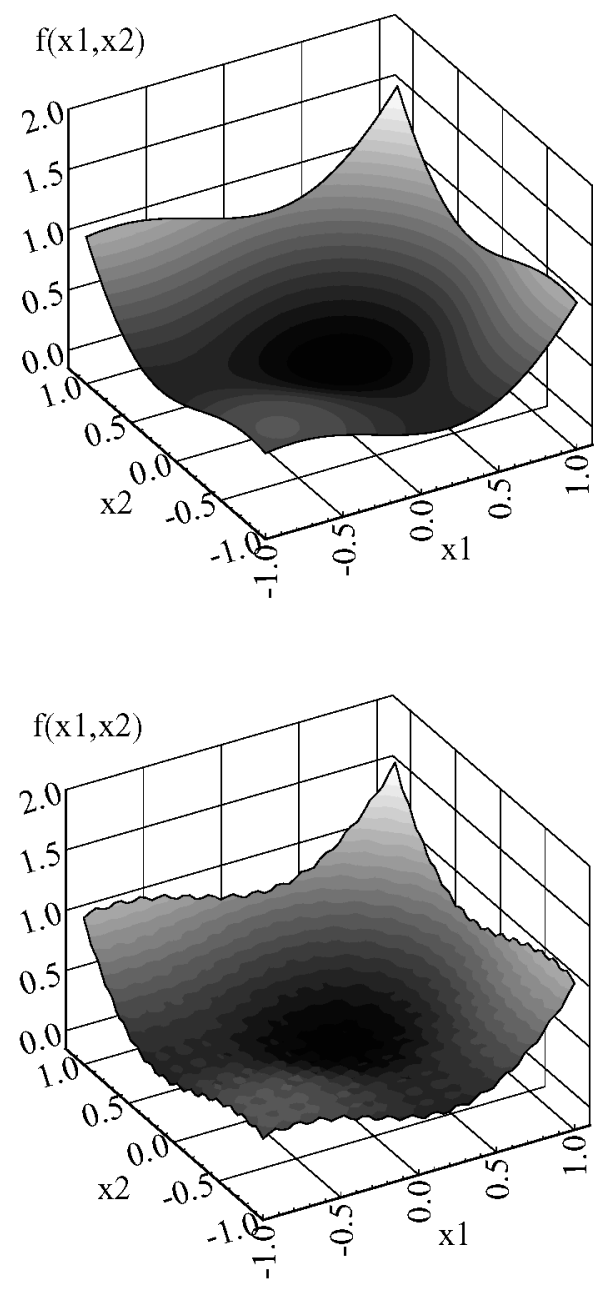

Figure 4: A two dimensional view of the smooth (top) and noisy variants of the Case 2 test function $(\epsilon=$ $0.7)$. 


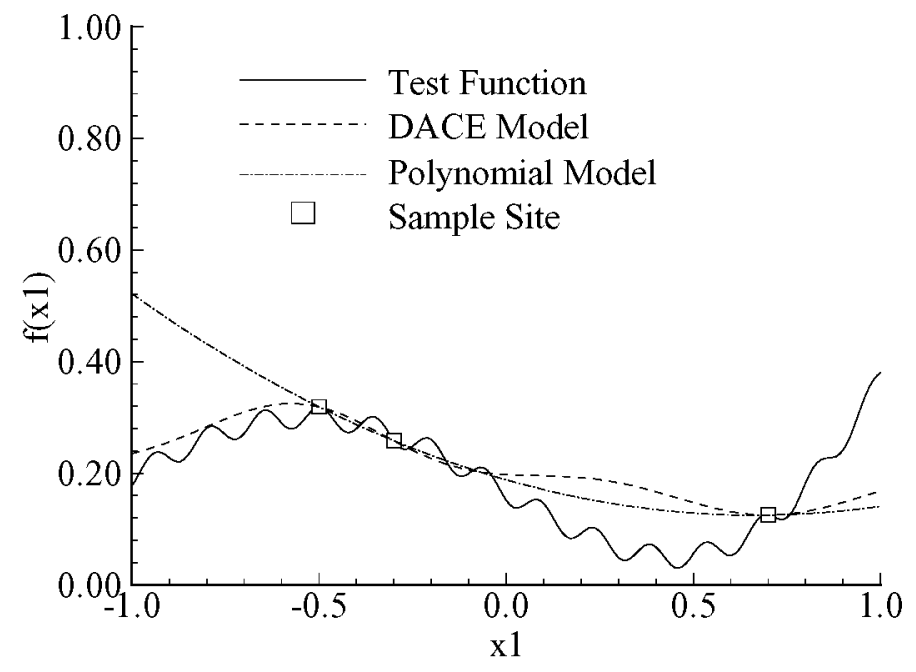

Figure 5: The DACE and quadratic polynomial RS models for Case $1\left(\begin{array}{lll}\epsilon & 1\end{array}\right)$ of the one variable test

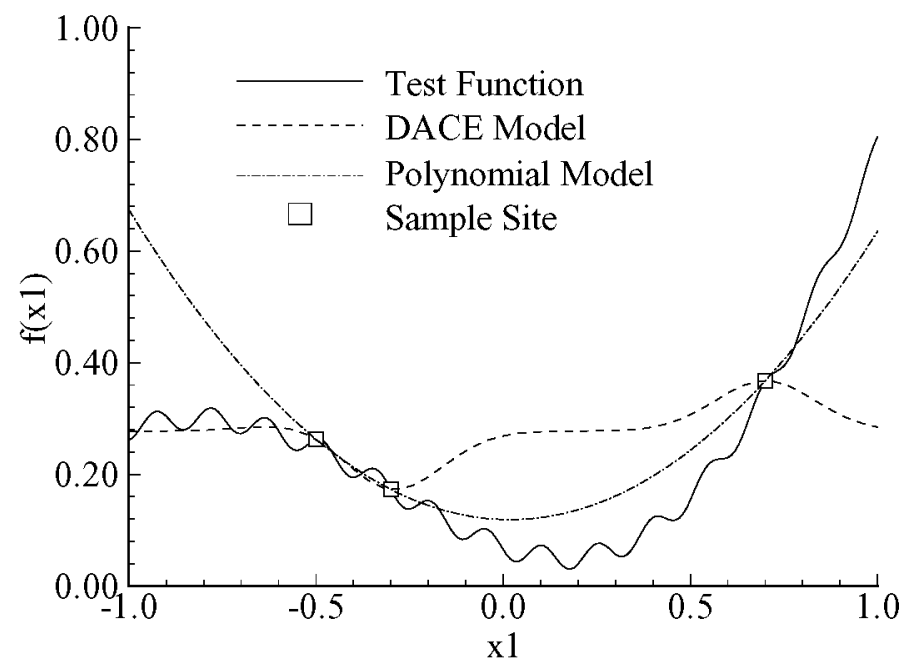

Figure 6: The DACE and quadratic polynomial RS models for Case $2(\epsilon=0.7)$ of the one variable test function. 